\title{
Zur Kenntnis der Gattung Molops (Coleopt., Carab.).
}

(Mit besonderer Berücksichtigung der in Albanien und Montenegro vorkommenden Arten und Rassen.)

Von Dr. Josef Müller (dz. in Wien).

(Schluß.)

Diese Rasse ist in Größe und Gestalt dem serbischen $M$. Reiseri in hohem Grade ähnlich, sie unterscheidet sich jedoch von diesem durch etwas schwächere Ausbuchtung vor den Hinterecken des Halsschildes, fast geraden Basalrand der Flügeldecken und vor allem durch die knopfartige Verdickung des Nahtwinkels, welche ihre nähere Verwandtschaft mit Holdhausi bekundet. Auch ist der Apikalteil des Penis wie bei letzterem hinter der großen Präputialöffnung plötzlich verschmälert, während bei Reiseri der Apikalteil des Penis an der Basis breiter und daher vom Präputialteil des Penis weniger scharf abgesetzt erscheint. Bei seitlicher Betrachtung ist der Penis von durmitorensis nur graduell von jenem des Reiseri durch etwas deutlichere doppelte Schwingung verschieden.

10. M. Victoris spec. nov.

Glänzend, schwarz (inklusive Beine). Der Kopf von normaler Größe, mit ziemlich geraden Stirnfurchen. Der Stirnrand über der Fühlerwurzel verdickt aber nicht nach außen vorspringend. Der Halsschild etwas schmäler als die Flügeldecken, quer, in der vorderen Hälfte schwach gerundet, nach hinten fast geradlinig verengt, die sehr kleinen, vom Seitenrand winkelig abgesetzten Hinterecken nicht scharfspitzig, jedoch nach außen deutlich vorspringend. Die Seitenrandung des Halsschildes mäßig dick, hinten kaum breiter als vorne; die beiden Basalstriche ziemlich lang und fast gerade nach vorne gerichtet, der zwischen dem äußeren und inneren Basalstrich befindliche schwach gewölbte Zwischenraum in der Mitte der Quere nach vertieft. Die Flügeldecken nicht ganz anderthalbmal so lang als breit, in der Mitte am breitesten, an den Seiten gleichmäßig schwach gerundet, an der Basis erheblich breiter als die Halsschildbasis. Der Basalrand 
der Flügeldecken fast gerade, die Schultern eckig, der SchulterwinkeI etwas nach außen vortretend. Der Apikalrand der Flügeldecken nach innen kurz abgeschrägt, so daß die Flügeldeckenspitze am Nahtende einen stumpfen einspringenden Winkel aufweist, der jederseits von einem stumpfwinkeligen Vorsprung des Apikalrandes begrenzt wird. Die Flügeldeckenstreifen mäßig stark, die Zwischenräume ziemlich flach; der siebente Zwischenraum vorne etwas breiter als der sechste, der achte schmal, auch vorne nicht breiter als der neunte. Die Punkte der Series umbilicata ziemlich klein, in der Mitte viel spärlicher als gegen die Basis oder gegen die Spitze. Die Hinterschienen auf der Dorsalkante mit zwei Sägezähnchen, von denen je eine kleine Borste entspringt. Der Penis hinter der Präputialöffnung eingeengt mit langer, ziemlich scharfer, etwas nach rechts gekrümmter Spitze; von links betrachtet erscheint der Apikalteil des Penis nur äußerst schwach doppelt geschwungen, die Spitze ist nur äußerst schwach abwärts gekrümmt (am ehesten mit Fig. 11 in Apfelbecks Käferfauna der Balkanhalbinsel I, 219 übereinstimmend). Länge $13 \mathrm{~mm}$, Breite $5 \mathrm{~mm}$.

Fundort: Prokletija-Gebirge an der albanesisch-montenegrinischen Grenze, 1300 - 2000 m Höhe. Vom Herrn Kustos Dr. Arnold Penther 1914 entdeckt (l $\left.\begin{array}{l}1 \\ 0\end{array}\right)$. Nach Herrn Regierungsrat Viktor Apfelbeck, dem wir wertvolle Studien über die Molops-Arten der Balkanhalbinsel verdanken, benannt.

Das + von $M$. Victoris glaube ich in einem Exemplar zu erblicken, das ebenfalls von Kustos Penther im albanesisch-montenegrinischen Grenzgebirge (Andrijevica, 31. V. bis 2. VI. 1914) erbeutet wurde. Es ist zwar erheblich größer als das ô vom Prokletija-Gebirge, auch ist der Halsschild nach hinten stärker verengt und vor den Hinterecken deutlich ausgeschweift; doch stimmt die Bildung der Flügeldeckenspitze (einspringender stumpfer Winkel am Nahtende) ganz auffällig mit $M$. Victoris überein. Länge $15,5 \mathrm{~mm}$, Breite $5,7 \mathrm{~mm}$.

M. Victoris gehört in die Verwandtschaftsgruppe des M. Reiseri, mit dem unsere Art auch im Penisbau am ehesten übereinstimmt; jedoch ist die serbische Art etwas kleiner, ihr Halsschild in beiden Geschlechtern von den Hinterecken deutlich ausgeschweift, der Basalrand der Flügeldecken stärker eingebuchtet, ihre Spitzen gemeinsam verrundet oder höchstens am Nahtwinkel etwas eingezogen, jedoch ohne den für Victoris charakteristischen, breitwinkeligen Einschnitt, schließlich der rechte Penisrand gegen den Apikalteil stärker eingebogen, der Apikalteil selbst dadurch bei Betrachtung von oben schmäler erscheinend. 
Von M. Holdhausi und dessen Rasse durmitorensis ist M. Victoris durch gänzlich verschiedene Bildung der Flügeldeckenspitze (Mangel der knopfartigen Verdickung am Nahtende), vom ersteren auch durch bedeutendere Größe, dunkle Beinfärbung und bei seitlicher Betrachtung viel schwächer doppelt geschwungenen Penis auffallend verschieden.

Von $M$. curtulus, dem unsere Art in Färbung, Größe und Habitus sehr ähnelt und mit dem sie auch im Prokletija-Gebirge zusammen vorkommt, unterscheidet sie sich durch den scharfspitzigen und bei seitlicher Betrachtung schwach doppelt geschwungenen Penis. Das Ende der Flügeldecken ist zwar bei curtulus fast immer etwas eingezogen, jedoch ist der Apikalrand jederseits des einspringenden Nahtwinkels breit verrundet, bei Victoris stumpfwinkelig vortretend.

Von den anderen in Nord-Albanien vorkommenden Molops-Arten wären nur noch albanicus und Sturanyi zum Vergleiche heranzuziehen. Beide haben einen zur Basis stärker verengten Halsschild und gemeinsam verrundete Flügeldeckenspitzen; ersterer ist auch durch die meist roten Beine und die stumpfere Penisspitze, letzterer durch ventralwärts stärker gekrümmte Penisspitze von $M$. Victoris leicht zu unterscheiden.

\section{M. Apfelbecki plurisetosus subspec. nov.}

Vom typischen M. Apfelbecki vom Volujak durch bedeutendere Durchschnittsgröße, stets dunkel gefärbte Beine und häufige Vermehrung der Supraorbital- und Marginalborsten des Halsschildes verschieden. Die hintere Supraorbitalborste ist nicht selten verdoppelt und statt der normalen vorderen Marginalborste des Halsschild€s sind deren häufig $2-3$ vorhanden. Im übrigen zeichnet sich diese Rasse durch eine abnorm starke Variabilität der Halsschildhinterecken aus. Dieselben sind meist groß und scharf rechtwinkelig oder etwas spitz; es kommen aber auch Stücke mit stark zahnförmig nach außen vortretenden Hinterecken vor. Bei einem Exemplar sind dieselben auffallend kleiner, etwa so wie bei Pareyssi, von dem es nur durch den am rechten Apikalrand schwach ausgebuchteten Penis abweicht. Länge 14,5-17 $\mathrm{mm}$.

Von Dr. Penther an mehreren Fundorten im albanesisch-montenegrinischen Grenzgebirge entdeckt: Prokletija, $1200-2000$ m, 1914; Rikavac, Juni 1914; Vermosa, 20.-21. VII. 1914. Úberall in Gesellschaft des $M$. montenegrinus, dessen dunkle Exemplare bei flüchtiger Betrachtung leicht mit der vorliegenden Rasse verwechselt werden können. Sicher gelingt aber die Unterscheidung durch In- 
spektion des Penis, der bei montenegrinus eine viel plumpere, breit verrundete Spitze besitzt.

Anmerkung: Das bisher zur Trennung von Molops und Stenochoromus benutzte Merkmal betreffend die Zahl der Marginalborsten des Halsschildes wird durch die Entdeckung des M. Apfelbecki plurisetosus hinfällig. Da auch die Beschaffenheit des Prosternalfortsatzes - ob umkantet oder nicht umkantet - bei der Untersuchung größerer Serien von $M$. montenegrinus im Stiche läßt, so fällt es schwer, die Untergattung Stenochoromus scharf zu definieren. Der eigenartige Habitus typischer montenegrinus-Exemplare unterliegt auch gewissen Schwankungen und wird von manchen Exemplaren der vorliegenden Apfelbecki-Rasse täuschend nachgeahmt.

\section{M. piceus mehadiensis subspec. nov.}

Große, kräftige, schwarzbeinige Rasse, im weiblichen Geschlechte selbst kleine $M$. elatus - Exemplare an Größe übertreffend. Vom typischen piceus aus dem deutschen Mittelgebirge auch durch weniger spitze Hinterecken des Halsschildes, stärker gestreifte Flügeldecken, vor der gekrümmten Spitze seichter ausgebuchteten rechten Penisrand sowie durch den Besitz von zwei Borsten auf der Dorsalkante der Hinterschienen differierend. Die Flügeldecken des $q$ wie beim echten piceus gestaltet, tief ausgeschnitten, außen durch einen starken winkeligen Vorsprung begrenzt. Länge 12-16 mm, Breite 4,8-5,8 $\mathrm{mm}$.

Fundort: Mehadia (Herkulesbad) im Banat. Von weiland Regierungsrat L. Ganglbauer gesammelt. Ebenda auch $M$. austriacus in einer besonderen Rasse (orthogoni's Chaud.), ohne daß ich bisher Übergangsstücke gesehen hätte. Wie ich in meiner ausführlicheren Arbeit beweisen werde, sind $M$. austriacus und piceus bei Mehadia in beiden Geschlechtern verschieden und können daher nicht ohne weiteres identifiziert werden, wie es in neuerer Zeit von mancher Seite geschehen ist.

\section{M. austriacus tridentinus subspec. nov.}

Vom echten austriacus aus Nieder-Österreich und Steiermark durch bedeutendere Größe, schwarze Beine, stärker gestreifte Flügeldecken, spitzige, nach außen vorspringende Hinterecken des Halsschildes und längeren, stärker gekrümmten Apikalteil des Penis verschieden. Auch haben die Hinterschienen fast immer auf ihrer Dorsalkante zwei abstehende Börstchen, während der echte austriacus normal deren nur eine besitzt. Länge $11-15 \mathrm{~mm}$, Breite 4,3-5,4 mm. 
Diese Rasse bewohnt das Gebiet der Lessinischen Alpen südlich von Rovereto: Vallarsa (leg. Winkler); Mandriola und Piano della Fugazza (leg. Ganglbauer).

\section{M. austriacus longulus subspec. nov.}

Vom typischen austriacus Gglb. durch viel gestrecktere Körperform: schmäleren Halsschild und viel längeren und schmäleren gleichmäßig verjüngten, am Ende verrundeten Apikalteil des Penis verschieden. Die Hinterecken des Halsschildes rechtwinkelig oder etwas abgestumpft, der innere Basalstrich zwar tief, aber kürzer als bei austriacus, der Basalrand des Halsschildes an den Hinterecken deutlich abgeschrägt. Beine rötlich braun, Hinterschienen auf der Dorsalkante mit einem Börstchen. Länge 10,5-13 mm, Breite 3,5-4,2 mm.

Fundort: Troglav-Gebirge, in den Dinarischen Alpen an der bosnisch-dalmatinischen Grenze. Vom Herrn A. Winkler (Wien) 1914 gesammelt.

Stücke von der Klekov، ča planina in N.-W.-Bosnien stimmen in der Körperform und in der Länge der Penisspitze am ehesten mit austriacus longulus überein; nur ist der Halsschild etwas breiter, namentlich an der Basis, und die Hinterecken desselben sind schärfer rechtwinkelig, bisweilen etwas spitzig.

\section{Bestimmungstabelle der mir bekannten Molops-Arten und -Rassen aus Albanien und Montenegro.}

Durch obige Neubeschreibungen sowie durch die neueren Arbeiten Apfelbecks über die Gattung Molops hat besonders die albanesischmontenegrinische Fauna eine Bereicherung erfahren. Während noch in der letzten zusammenfassenden Bearbeitung der Gattung (Apfelbeck, Käferfauna der Balkanhalbinsel I, 216-233) 10 Molops-Arten aus Albanien und Montenegro behandelt werden, ist die Zahl nunmehr auf 18 Arten und 5 Rassen gestiegen. Es dürfte daher nachfolgende Bestimmungstabelle der albanesisch-montenegrinischen Molops-Arten und Rassen nicht unerwünscht sein. Eine Bearbeitung sämtlicher europäischer Formen hoffe ich binnen kurzem - nach Einsichtnahme einiger mir fehlender Typen — fertigstellen zu können.

1. Gewölbtere, meist schwarze Arten mit einem, seltener mit 2-3 Borstenpunkten in der Seitenrandkehle des Halsschildes vor der Mitte. Wenn daselbst mehr als eine Borste vorhanden (Apfelbecki plurisetosus), dann stehen dieselben näher beisammen und die vorderste ist stets von den Vorderecken weit entfernt. 
Der Prosternalfortsatz hinter den Vorderhüften nicht umkantet (subg. Molops s. str.) . . . . . . . . . . . . . . 2

— Sehr flache, meist rötlich oder pechbraune Art mit 3-5 Borstenpunkten in der Seitenrandkehle des Halsschildes vor der Mitte. Die Borsten sind fast über die ganze Apikalhälfte der Halsschildseiten verteilt, die vorderste Borste entspringt in der Nähe der Vorderecken. Der Prosternalfortsatz in der Regel umkantet (subg. Stenochoromus Mill.)

2. Nahtwinkel der Flügeldecken knotig verdickt und vorspringend. Der Halsschild zur Basis schwach verengt, an den winzigen, nach außen winkelig vorspringenden Hinterecken etwa so breit als am Vorderrand. Basalrand der Flügeldecken fast gerade 3

_ Nahtwinkel der Flügeldecken nicht knotig vorspringend. Der Falsschild zur Basis viel stärker verengt, daher an der Basis bedeutend schmäler

3. Kleiner und gedrungener, rotbeinig, die Halsschildseiten vor den Hinterecken nicht deutlich ausgeschweift; der Penis von links betrachtet stark doppelt geschwungen erscheinend. L. $10 \mathrm{~mm}$. - Prokletija-Gebirge

Holdhausi $\mathrm{m}$.

- Etwas größere und gestrecktere, dunkelbeinige Rasse. Die Halsschildseiten vor den Hinterecken etwas ausgeschweift; der Penis bei seitlicher Betrachtung mit einer schwächeren doppelten Schwingung. L. $12 \mathrm{~mm}$. - Durmitor-Gebirge

Holdhausi durmitorensis $\mathrm{m}$.

4. Flügeldecken am Ende mit einem gemeinsamen stumpfwinkeligen Einschnitt, der Apikalrand bildet jederseits einen stumpf verrundeten Vorsprung. - Dem M. curtulus ähnlich, jedoch ist der Penis scharf zugespitzt und bei seitlicher Betrachtung schwach doppelt geschwungen. L. $13-15,5 \mathrm{~mm}$. - Prokletija-Gebirge

Victoris $\mathrm{m}$.

- Flügeldecken am Ende ohne stumpfwinkeligen Einschnitt, höchstens das Nahtende schwach eingezogen, dann aber der Apikalrand jederseits gleichmäßig gerundet, ohne stumpfen Vorsprung

5. Der äußere Basalstrich des Halsschildes vom Seitenrande weiter abgerückt; der innere Basalstrich viel schwächer, bisweilen undeutlich. Falls ein solcher vorhanden, so steht er dem äußeren Basalstrich näher als dieser dem Seitenrande. Große, auf den Flügeldecken stark gestreifte Art mit ziemlich kurzer und gerader, breit verrundeter Penisspitze. L. $17-21 \mathrm{~mm}$. Úsküb und Schar-Dagh-Gebiet (Apfelbeck, XII. Jahres- 
bericht naturwiss. Orientvereins Wien, 1907, 33 und Sitzungsber. Akad. Wien, math.-naturw. Klasse, Bd. CXVI, Abt. 1, 1907, 503)

robustus Dej.

— Der äußere Basalstrich des Halsschildes dem Seitenrande stark genähert, von diesem durch einen schmäleren Zwischenraum als vom inneren Basalstrich getrennt .

6. Der Apikalteil des Penis ${ }^{1}$ ) sehr kurz, fast breiter als lang, breit verrundet. Breite und gewölbte Art mit feingestreiften Flügeldecken. Der 7. und 8. Zwischenraum meist sehr schmal, kielförmig, beide zusammen selten so breit, in der Regel schmäler als der 6. Zwischenraum. Die Punkte der Series umbilicata schmal und daher oft wenig auffällig. L. 14-18 mm. - Sutorman (Apfelbeck, Käf. Balk. I, 220), Maranai (Apfb., Sitzungsber. Akad. Wiss., 1907, 497) und Rapsa (Dr. Penther, V. 1914, 10 !)

simplex Chaud.2)

- Der Apikalteil des Penis länger und deutlicher verschmälert, fast immer deutlich länger als breit. Wenn größere und gewölbtere Arten, dann sind die Punkte der Series umbilicata viel größer und rund .

7. Apikalteil des Penis gerade gen $^{3}$ gegen die stumpf verrundete Spitze dreieckig verschmälert. Ziemlich flache Art mit spitzen oder rechtwinkeligen, meist kleinen, selten durch eine breitere Ausbuchtung des Seitenrandes länger erscheinenden Hinterecken des Halsschildes. L. $13-16,5 \mathrm{~mm}$. - N jeg $\mathrm{s} \check{\mathrm{s}}$ (Apfb. 1903, Wien. Hofmus.!), Sutorman (Apfb., Käf. Balk. I, 221) und Maranai (Apfb., Wien. Hofmus.!) . . . P Pareyssi Kr.

- Apikalteil des Penis mehr oder weniger deutlich nach rechts gekrümmt .

1) Ich bezeichne damit den ganzen hinter der Öffnung des Präputialsackes gelegenen Endabschnitt des Penis. Für das distale, häufig charakteristisch geformte Ende des Apikalteiles gebrauche ich den Ausdruck ,Penisspitze".

2) Äußerst nahe verwandt ist der nach Apfelbeck auch in SüdAlbanien (Valona - Dr. Patsch) vorkommende griechische M. parnassicola $\mathrm{Kr}$. Von $M$. simplex durch nach hinten stärker verengten Halsschild, kürzeren, äußeren Basalstrich desselben und im männlichen Geschlechte deutlich abwärts gekrümmte Penisspitze versêhieden.

${ }^{3}$ ) Vgl. evt. auch den durch die stets lang abgesetzten Hinterecken des Halsschildes charakterisierten, im übrigen aber mit Pareyssi äußerst nahe verwandten $M$. Apfelbecki, ferner den durch die stärker zugespitzten Flügeldecken und den viel schlankeren, doppelt geschwungenen Penis eicht unterseheidbaren M. albanicus. 
8. Apikalteil des Penis bei seitlicher Betrachtung deutlich doppelt geschwungen .

- Apikalteil des Penis bei seitlicher Betrachtung mehr oder weniger stark ventralwärts gekrümmt, jedoch nicht doppelt geschwungen

9. Mittelgroße, meist rotbeinige Art von Pareyssi-Habitus. Halsschild nach hinten stärker verengt; Flügeldecken gegen das Ende etwas zugespitzt, der Schulterwinkel stumpfer. L. 13,2-14,3 mm. - Prizren, Schar-Dagh-Gebiet, zusammen mit $M$. rufipes und osmanilis (Apfb., Käf. Balk. I, 223); M. Schèit, Merdita (Apfb., April-Mai 1905, Wien. Hofmus.!); Zebia Merdita (Apfb., Wien. Hofmus.!) . . . albanicus Apfb.

- Kleinere, dunkelbeinige Art von curtulus-Habitus. Halsschild nach hinten schwach verengt, an der Basis viel breiter; Flügeldeckenspitze breit vermehrt, der Schulterwinkel besonders beim ô schärfer markiert. L. 10,5-12 mm. - Serbisch-türkisches Grenzgebiet in der Umgebung von Novipazar: KopaonikGebirge und Raška (Reiser 1899, Wien. Hofmus.!)

Reiseri Apfb.

10. Apikalteil des Penis stark herabgekı ümmt; bei seitlicher Betrachtung erscheint die Krümmung der Ventralfläche hinter der Präputialöffnung plötzlich stärker .

- Der Penis schwach und gleichmäßig ventralwärts gekrümmt; bei seitlicher Betrachtung erscheint die Krümmung hinter der Präputialöffnung nicht stärker als vorher .

11. Der innere Basalstrich des Halsschildes kräftig entwickelt, stark vertieft, der äußere dagegen viel schwächer, bisweilen fast ganz obliteriert. Hinterecken des Halsschildes ziemlich lang, spitzig. L. $11-13$ mm. - Ljubeten, Schar-Dagh (Apfb., Wien. Hofmus.!)

Steindachneri Apfb.

- Der äußere Basalstrich des Halsschildes fast ebenso stark vertieft als der innere

12. Häufig rotbeinige Art von etwas schlankerem Habitus. Halsschild nach hinten in schwächerer Kurve verengt, vor den Hinterecken länger ausgeschweift, seine Maximalbreite vor der Mitte gelegen .

- Dunkelbeinige, gedrungenere Art. Halsschildseiten stark und fast gleichmäßig gerundet, vor den Hinterecken nicht oder nur schwach ausgeschweift, die Maximalbreite etwa in der Mitte 
gelegen. Länge 11,5-13 mm. - Bulgarien: Stara planina bei Klisura (Merkl, Wien. Hofmus.!), aber auch bei R ǎ̌ka an der serbisch-türkischen Grenze in der Umgebung von Novipazar (Apfb., Käf. Balk. I, 222) . . . . . . . klisuranus Apfb.

13. Größer, $13-14 \mathrm{~mm}$ lang. Apikalteil des Penis etwas schwächer abwärts gekrümmt. - Zebia, Merdita (Wien. Hofmus.!); Mal i Shêit, Merdita (Apfb., Sitzungsber. Akad. Wiss. Wien, math.-naturw. Klasse, CXV, Abt. 1, 1906, 1665); Maranai, Montenegro (Apfb., Sitzungsber. Akad. Wiss. 1907, 497)

Sturanyi Apfb.

-_. Etwas kleinere, 11,3 - 13 mm lange Form mit sehr stark ventralwärts gekrümmter Penisspitze. - Munela, Merdita (Winneguth, Wien. Hofmus.!) . . . . . subspec. munelensis Apfb.

14. Die Penisspitze mehr oder weniger gedreht und in einiger Entfernung von der Spitze am rechten Penisrand mit einem klein€n zahnförmigen Vorsprung versehen. Kleine Art mit ovalen Flügeldecken und relativ breitem 7. Zwischenraum derselben; Hinterecken des Halsschildes groß und scharf. Länge 10-12 mm. - Raspa und Prokletija-Gebirge im montenegrinischalbanesischen Grenzgebiet (Dr. Penther 1914, Wien. Hofmus.!) Umgebung von Úsküb am Fuße des Schar-Dagh (Apfelbeck, Wien. Hofmus.!) . . . . . . . . . austriacus subspec.

— Penisspitze nicht gedreht, ohne zahnförmigen präapikalen Vorsprung .

15. Penis am Ende scharf zugespitzt. Große Arten mit seitlich stark gerundetem, zur Basis stark verengtem Halsschild; die Hinterecken desselben klein, beim $q$ meist stumpf. Länge $17-19 \mathrm{~mm}$. . . . . . . . . . . . . . . . 16

- Penis am Ende mehr oder weniger verrundet oder abgestumpft 17

16. Die äußeren Basalstriche des Halsschildes etwas stärker entwickelt, nach vorne parallel oder divergierend; der Zwischenraum zwischen äußerem und innerem Basalstrich deutlicher vertieft. Die Flügeldecken namentlich beim o breiter und gewölbter. Der Apikalteil des Penis in der Dorsalansicht etwas breiter und weniger scharf zugespitzt. - Podgorica, montenegrinisch-albanesisches Grenzgebiet

obtusangulus intermedius $\mathrm{m}$.

— Die äußeren Basalstriche des Halsschildes schwächer entwickelt, nach vorne konvergierend, die inneren sehr lang und tief; der Zwischenraum zwischen äußerem und innerem Basalstrich kaum 
vertieft oder einfach gewölbt. Flügeldecken flacher und schmäler mit vorne stark aufgebogenen Seitenrändern. Der Apikalteil des Penis schmäler und schärfer zugespitzt. - Merdita: Mal i Shêit bei Oroshi und Zebia-Gebirge bei Fandi (Apfb., Wien. Hofmus.!). - Wahrscheinlich nur als eine extreme Rasse des $M$. obtusangulus aufzufassen

merditanus Apfb.

17. Halsschild mit kleinen Hinterecken. Gewölbtere oder wenigstens kürzere Arten .

- Halsschild mit großen recht- oder spitzwinkeligen Hinterecken. Gestreckte Art mit flachen Flügeldecken

18. Kräftige, gewölbte Arten mit breit verrundeter und stark nach rechts gekrümmter Penisspitze. Die Halsschildseiten meist bis zu den kleinen Hinterecken gerundet. Die Mittelschienen des ô ziemlich stark nach innen gekrümmt .

- Kleinere, flachere Art mit schmal verrundeter und nur schwach nach rechts gewendeter Penisspitze. Die Halsschildseiten vor den Hinterecken meist etwas ausgeschweift. Flügeldecken am Ende fast immer einzeln gerundet mit schwach einspringendem Nahtwinkel. Länge $13-15 \mathrm{~mm}$. - Maranai, Montenegro (Apfb., Sitzungsber. Akad. Wiss. 1907, 497); Durmitor (Dr. Penther 1901, 1 Ex.!). Prokletija-Gebirge, Rapsa, Grebeni, Vermosa und Rikavac im albanesisch-montenegrinischen Grenzgebiet (Dr. Peuther 1914!) . curtulus Ganglb.

19. Meist größere, breitere und stärker gewölbte, dunkelbeinige Formen. Die Halsschildseiten bis zu den sehr kleinen, bisweilen stumpfen Hinterecken in gleichmäßiger starker Rundung verengt

- Durchschnittlich kleinere, schmälere und flachere, oft rotbeinige Art. Die Halsschildseiten nach hinten in schwächerer Kurve verengt, vor den in der Regel sehr stark nach außen vortretenden spitzen Hinterecken geradlinig oder etwas ausgeschweift. Länge 12,5-16 $\mathrm{mm}$. - Vlasulja, Volujak im montenegrinischherzegowinischen Grenzgebiet, hochalpin an Schneefeldern (Apfelbeck 1890, Speiser 1892, Wien. Hofmus.!). In Süd-Bosnien (z. B. Romanja-planina bei Sarajevo, leg. Penther!) zusammen mit alpestris jedoch vorwiegend in höheren Lagen

vlasuljensis Ganglb.

20. Größere Form von obtusangulus-Habitus. Der Halsschild nach hinten stärker verengt mit schmälerer Basis, die Hinterecken recht- oder stumpfwinkelig. Flügeldecken stärker gestreift. 
Länge 17,5-18 mm. - Rapsa, albanesisch-montenegrinische Grenze $^{1}$ )

alpestris imitator $\mathrm{m}$.

- Kleinere, auf den Flügeldecken viel feiner gestreifte Rasse. Der Halsschild nach hinten weniger verengt mit breiterer Basis, die Hinterecken meist recht- oder stumpfwinkelig, selten spitz nach außen vortretend. Länge 14-16 mm. - Ljubeten, Schar-Dagh (M. alpestris rhilensis Apfb. pars)

alpestris ljubetensis $\mathrm{m}$.

21. Kleinere, meist rotbeinige Form mit zwei Supraorbital- und einer vorderen Marginalborste am Halsschild. Länge 13,5 bis $15 \mathrm{~mm}$. - Volujak (Apfb. und Speiser, Wien. Hofmus.!)

Apfelbecki Ganglb.

- Größere, stets dunkelbeinige Form mit häufiger Verdoppelung der hinteren Supraorbitalborsten und Vermehrung der Seitenrandborsten $(2-3)$ in der vorderen Hälfte des Halsschildes. Länge 14,5-17 mm. - Prokletija, Rikavac und Vermosa im albanesisch-montenegrinischen Grenzgebiet

Apfolbecki plurisetosus m.

22. Größere und breitere, pechschwarze Form aus dem westl chen Montenegro. Prosternalfortsatz in der Regel scharf umkantet. Länge 16,5-18 mm. - Njegus, Lovč ngebiet (Apfb., Wien. Hofmus.!)

montenegrinus Mill.

- Kleinere, schmälere und selbst in ausgefärbten Stücken hellere, pechbraune oder rotbraune, hochalpine Form vom Volujak (Apfb., Wien. Hofmus.!). Länge $14-15$ mm

subspec. nivalis Apfb.

- Sehr große und breite, rostrote Rasse. Noch heller als nivalis gefärbt, viel größer und breiter als dieser. Die Hinterecken des Halsschildes deutlich spitz nach außen vortretend; die Flügeldecken breit, der Seitenrand gegen die Schulterecke stärker eingezogen, der achte Zwischenraum breiter und nicht deutlich kielförmig. Länge $18-20 \mathrm{~mm}$. - In der Höhle Sckamit dusckut am Mte. Cukali bei Skutari unter tief eingebetteten Steinen (Ent. Blätt. 1916, 81) . . . . subspec. cavernicola m.

Zahlreiche mir vorliegende Exemplare aus dem albanesischmontenegrinischen Grenzgebiet (Prokletija, Rikavac und

1) Unbekannt sind mir die alpestris-Stücke, die Apfelbeck von anderen montenegrinischen Fundorten anführt (Maranai, Sitzungsber. Akad. Wiss. 1907, 497; Durmitor und Kolašin, Käf. Balk. I, 224), ebenso die alpestris-Form von Üsküb am Fuße des Schar-Dagh (vgl. Apfb., XII. Jahr Ber. naturw. Orientvereins, Wien 1907, 33). 


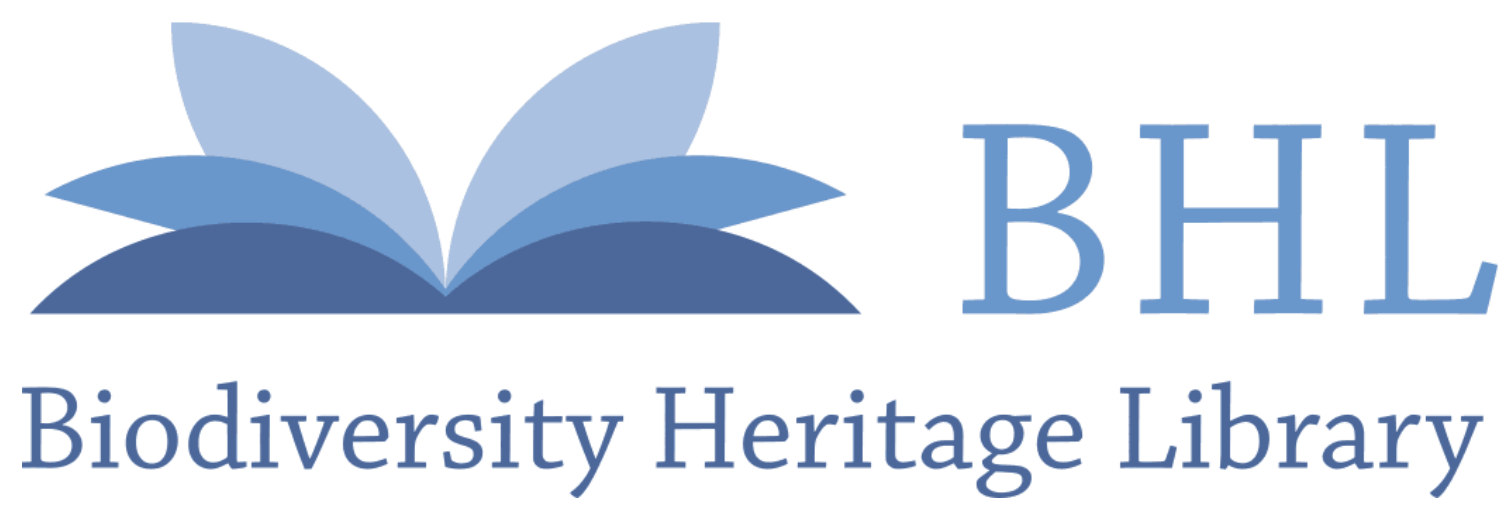

1918. "Zur Kenntnis der Gattung Molops." Entomologische Mitteilungen 7, 1-11. https://doi.org/10.5962/bhl.part.27257.

View This Item Online: https://www.biodiversitylibrary.org/item/40402

DOI: https://doi.org/10.5962/bhl.part.27257

Permalink: https://www.biodiversitylibrary.org/partpdf/27257

\section{Holding Institution}

Smithsonian Libraries

\section{Sponsored by}

Smithsonian

\section{Copyright \& Reuse}

Copyright Status: NOT_IN_COPYRIGHT

This document was created from content at the Biodiversity Heritage Library, the world's largest open access digital library for biodiversity literature and archives. Visit BHL at https://www.biodiversitylibrary.org. 